\title{
THE DFM CONTROL SYSTEM BASED ON PLL
}

A specific structure of control system with double fed machine (DFM) is studied in the paper. The DFM is assumed to be a generator with stator winding connected to the power grid and it produces active and reactive powers. A command vector controlling DFM is transformed to the rotor-oriented coordinate system using angle of transformation. The angle of transformation is estimated in the system by applying a phase locked loop (PLL).

Moreover, an example of application of pulse phase locked loop for estimation of voltage vector components is presented in the paper. In conclusion the properties of proposed PLL-based control systems are compared with properties of systems known from radio engineering and telecommunications described in the first chapter of the paper.

\section{Introduction}

The doubly fed induction machine (DFM) is suitable for generating systems working with variable rotor velocity. Shaft generators on ship and wind power systems are examples of application of DFM.

A specific structure of control system with DFM is studied in this paper. The DFM is assumed to be a generator with stator winding connected to the power grid and it produces active and reactive powers. The current regulated voltage source inverter (CRVSI) supplies the rotor winding. A command vector controlling DFM is transformed to rotor-oriented coordinate system using angle of transformation. The angle of transformation is estimated in the system with application of a phase locked loop (PLL). An application of PLL in considered control system allows omitting rotor position sensor (encoder). Examples of application of PLL to estimate the angle of transformation are given in papers [1,2]

The phase feedback known from radio engineering and telecommunications as phase locked loop (PLL) is conventional solution

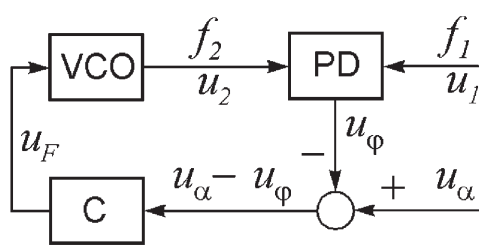

Fig. 1 The block diagram of circuit with PLL [3], where VCO is voltagecontrolled oscillator, $P D$ is phase detector, $C$ is controller, $u_{\varphi}$ is $P D$ output voltage (controlled voltage), $u_{\alpha}$ is reference voltage, $u_{\alpha}-u_{\varphi}$ is control error, $u_{F}$ is command voltage (control voltage), $f_{l}$ is standard frequency applied for synchronization of single-phase periodical signals [3, 4]. PLL equalizes frequency of voltage-controlled oscillator (VCO) with standard frequency. It is useful e. g. in demodulation of frequency-modulated signal (FM) as well as in frequency multiplication.

The block diagram of the circuit with PLL is depicted in Fig. 1. The control voltage $u_{F}$ changes VCO output frequency in the neighbourhood of rest frequency $f_{0}$. Phase detector gives output voltage dependent on phase shift $\varphi$ between VCO output voltage $\left(u_{2}\right)$ and standard voltage $\left(u_{1}\right)$. The specific feature of the circuit shown in Fig. 1 is astatic character of controlled system (VCO together with PD). Thus, the phase shift $\varphi$ increases proportionally to time without limit if frequency $f_{2}$ differs from the standard frequency $f_{1}$. Therefore, a difference in frequency should approach zero.

The block diagram of PLL with application of multiplier as a phase detector is depicted in Fig. 2. The output voltage of the multiplier contains a high-frequency component and a low-frequency component. Abovementioned high-frequency is equal to the sum of basic frequencies $f_{1}$ and $f_{2}$, whereas low-frequency is equal to difference of both basic frequencies.

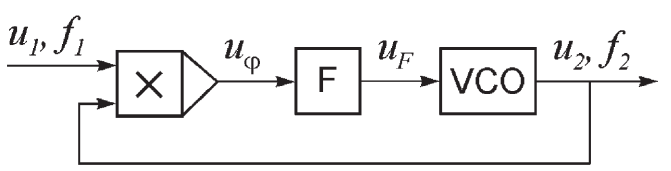

Fig. 2 The block diagram of PLL with multiplier as phase detector [4]

\footnotetext{
* Andrzej Popenda

Institute of Industrial Electrical Engineering, Faculty of Electrical Engineering, Technical University of Czestochowa, Poland, E-mail: popenda@el.pcz.czest.pl
} 
A low-pass filter $(\mathrm{F})$ is applied in order to eliminate the highfrequency component from PD output voltage. The output voltage of low-pass filter which controls VCO is expressed as follows:

$$
u_{F}=\frac{A V}{2} \cos \left[\int_{0}^{t}\left(\omega_{1}-\omega_{2}\right) d \tau-\varphi\right] .
$$

In steady state $\left(\omega_{1}=\omega_{2}\right)$ a value of voltage $u_{F}$ is constant:

$$
u_{F}=\frac{A V}{2} \cos \varphi \text {. }
$$

Operating range of PLL, i. e. allowable deviation of standard frequency from rest frequency $\left(\Delta f=f_{1}-f_{0}\right)$ for which the circuit shown in Fig. 2 still reaches synchronism, is limited by maximal and minimal value of voltage $u_{F}$. As it results from dependence (2), in the circuit shown in Fig. 2 mentioned values are determined for arguments $\varphi$ equal to 0 and $\pi$ while the rest value of angle $\varphi$ amounts to $\pi / 2$.

The phase shift between two voltages may also be detected through sample-and-hold circuit sampling actual value of voltage $u_{1}$ in the moments when voltage $u_{2}$ crosses the zero level in direction of positive values. The block diagram of PLL with S\&H circuit as phase detector as well as transient responses of the PLL explaining principle of operation are shown in Fig. 3 and 4.

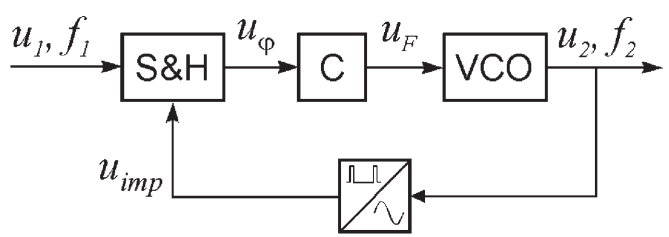

Fig. 3 The block diagram of PLL with $S \& H$ circuit as phase detector [3]

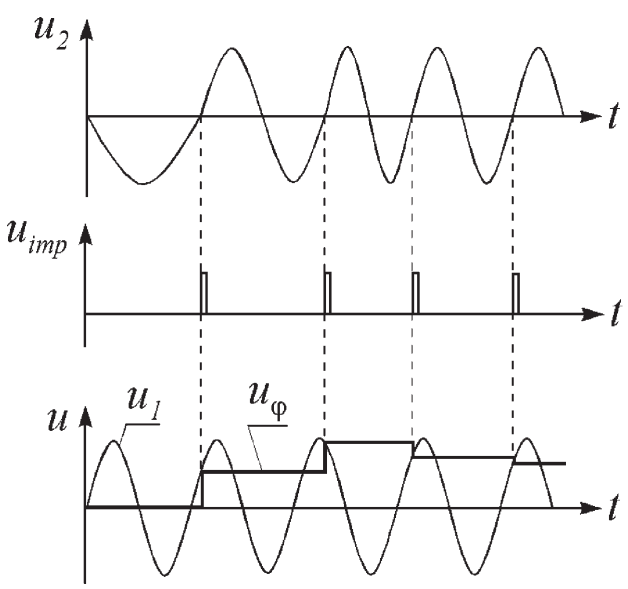

Fig. 4 Transient responses of the system shown in Fig. 3

Operating range of PLL depends on amplification in main circuit of considered structure working with unlocked loop (ampli- fication of input-actuated control system). There is no possibility of unlimited increase in value of amplification due to decrease of stability margin of the PLL. Therefore, operating range of PLL shown in Fig. 3 is not large (a few percent in comparison to the rest frequency $f_{0}$ ).

\section{PLL-Based Synchronization of Inverter Current in DFM Control System}

An application of nonlinear element (multiplier) as a phase detector causes distortion of the output signal $\left(u_{\varphi}\right)$. The low-pass filter is applied in order to decrease the distortion. The application of the low-pass filter causes a slower operation of PLL. A control of spatial vector position angle is used in poly-phase systems instead of synchronization of single-phase signals. A filtration of the angle calculated with application of vectors' calculus is unnecessary. Controlled variable $\left(u_{\varphi}\right)$ obtained as vectors' calculus product is proportional to the angle $\varphi$. Thus, maximal and minimal values of the control variable $\left(u_{F}\right)$ are determined by arguments $\varphi= \pm \pi$, whereas the rest value of $u_{F}$ appears when $\varphi=0$ provided that the reference variable $\left(u_{\alpha}\right)$ is equal to zero. The control systems with electric machines based on vector methods are examples of polyphase (three-phase) systems.

From the above analysis it follows that operating range of PLL in the poly-phase systems is wider than in the single-phase system shown in Fig. 2. Moreover, the phase shift $\varphi$ between $u_{1}$ and $u_{2}$ is equal to zero in a steady state when the PI controller is applied. A block diagram of DFM control system with PLL is depicted in Fig. 5. An application of PLL allows adjusting the frequency of the inverter current depending on power grid frequency and rotational speed of DFM rotor.

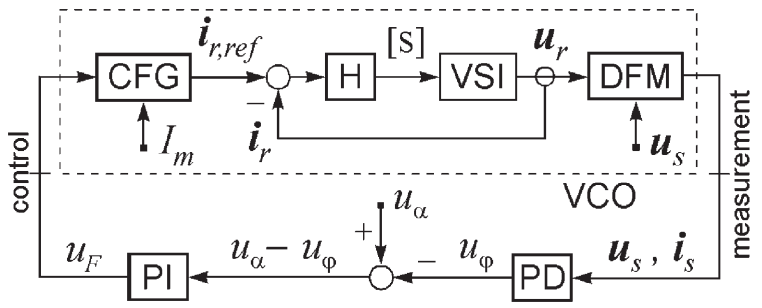

Fig. 5 The block diagram of DFM control system with application of $P L L$, where CFG is generator of current frequency, $H$ are current controllers, VSI is voltage source inverter, PI is angle controller,

$u_{s}$ is stator voltage vector, $i_{s}$ is stator current vector, $u_{r}$ is rotor voltage vector, $i_{r}$ is rotor current vector, $[S]$ is vector of command signals controlling the inverter switching

The DFM working with stator winding connected to the power grid and rotor winding fed via a current regulated voltage source inverter (CRVSI - locked circuits containing VSI together with controllers $\mathrm{H}$ ) is considered as a controlled system (plant). The current controllers $(\mathrm{H})$ allow keeping phase currents of the 
rotor in a required range. A generator of current frequency $(\mathrm{CFG})$ provides the current controllers with set values of controlled currents. Nonlinear CFG transforms set amplitude and set frequency to actual set currents in the three-phase system. The angle controller (PI) produces the set frequency of rotor current, while the phase detector (PD) produces a signal proportional to the angle $\varphi$ between the rotor current vector in stator terms and the stator voltage vector. A phase shift $\varphi$ as well as the ratio of active power $p$ and reactive power $q$ generated to the grid depend on the actual value of reference voltage $u_{\alpha}$.

A simple dependence may be used for approximation of the angle $\varphi[1,2]$ :

$$
\varphi=\operatorname{arctg} \frac{q-q_{0}}{p}+n \pi, n=-1,0,1,-\pi \leq \varphi \leq+\pi
$$

where $p=u_{s \alpha} i_{s \alpha}+u_{s \beta} i_{s \beta}, q=u_{s \beta} i_{s \alpha}+u_{s \alpha} i_{s \beta}$ are active power and reactive power of the stator, $q_{0} \approx u_{s}^{2} / \omega_{s} L_{s}$ is reactive power connected with magnetizing current of the induction machine. The application of the above dependence allows skipping a transformation of rotor current to the coordinate system connected to the stator voltage vector. Thus, a rotor position sensor (encoder) is omitted in the structure of the considered DFM control system. This is the most valuable advantage resulting from the application of PLL in the DFM control systems.

In the system shown in Fig. 6, PLL replaces the encoder and estimates the rotor position angle. The DFM power $p, q$ controllers produces set components $i_{r p, r e f}, i_{r q, \text { ref }}$ of the rotor current vector. The components are transformed to the rotor-oriented coordinate system 0xy and control the CRVSI. Moreover, the components $i_{r p, r e f}, i_{r q, \text { ref }}$ allow calculating the reference angle $\alpha$. The controller of the angle $\varphi$ estimates difference $\omega$ in angular velocity between the rotor and stator voltage vector. The angle $\gamma$

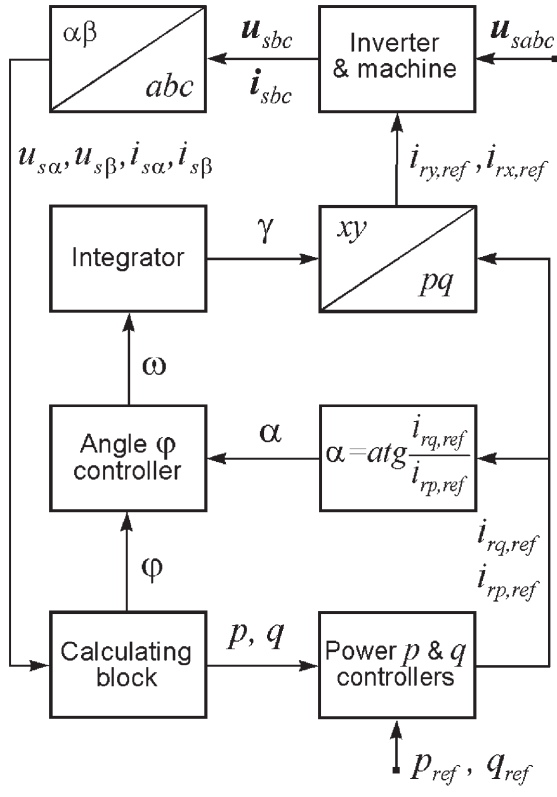

Fig. 6 The DFM power control system with PLL [2], where atg(.) is arc-tangent function

of rotor position in the coordinate system $0 \mathrm{pq}$ connected to the stator voltage vector is obtained as a result of integration of variable $\omega$ and allows transforming the set current components from $0 \mathrm{pq}$ to $0 \mathrm{xy}$ coordinate system.

Transient responses of the control system shown in Fig. 6 are given in Fig. 7. As it follows from the diagrams the considered system with PLL (Fig. 6) controls the active power $p$ and reactive power $q$ in short time without coupling between $p$ and $q$, similarly to the DFM control systems with an encoder $[1,5]$.
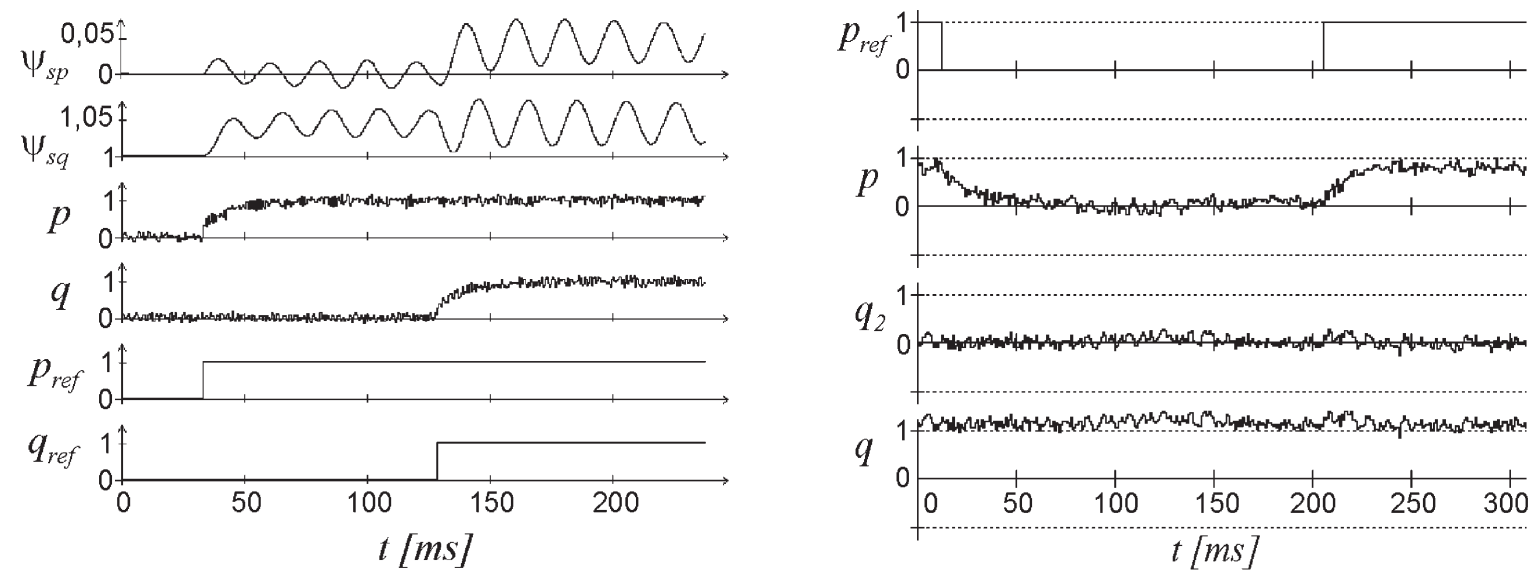

Fig. 7 Transient responses of control system shown in Fig. 6 obtained from digital simulations (left diagram) and experimental investigations (right diagram), where $p, q$ are active and reactive power generated to the power system from DFM stator winding, $p_{\text {ref }}, q_{\text {ref }}$ are reference values of active and reactive power, $q_{2}$ is reactive power of rotor, $\psi_{s p}, \psi_{s q}$ are components of stator flux vector in coordinate system connected to the stator voltage vector; the variables are given in non-dimensional units 
In designing the supply systems for electrical machines and electrical equipment it should not be omitted that the machines and equipment exert an influence on power grid and local electric power systems. The mains voltage drops and oscillations as well as the harmonics generated by electric machines and other disadvantageous phenomena from power quality point of view occur as a result of this influence [6 - 9]. The author of [10] pays attention to mentioned phenomena and he proposes a number of predicting analyses concerning power demand for individual consumers or groups of consumers. He also proposes predicting analyses concerning areas connected with work of selected sets of local consumers in power systems.

\section{An Estimation of Spatial Vector Position Using Pulse PLL}

The author used the pulse phase locked loop depicted in Fig. 8 to estimate the phase of stator voltage (power voltage) during experimental investigations. Estimated phase of stator voltage allows obtaining the components of stator voltage vector in an immovable coordinate system when the voltage amplitude is known.

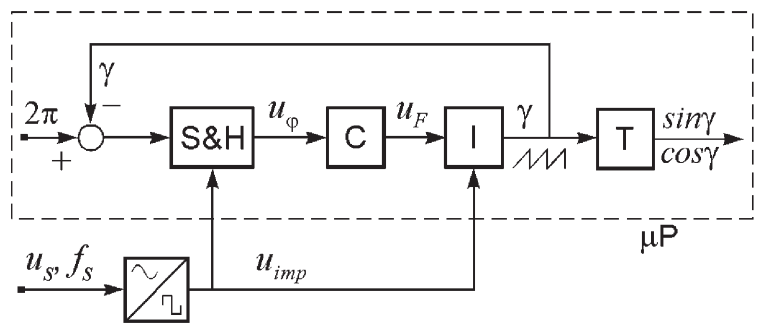

Fig. 8 The system with pulse PLL estimating phase of stator voltage, where $S \& H$ is sample-and-hold circuit, I is integrator, T are look-up tables of sine and cosine as a function of angle $\gamma, \mu P$ is microprocessor system

The advantages resulting from the application of pulse PLL are as follows. Only one binary input is required to load the pulses of voltage $u_{i m p}$ into a microprocessor system instead of two analogue inputs. Moreover, time-dependences of the estimated stator voltage vector components are undistorted in steady state.

A principle of system operation is described below. Quasi-sinusoidal single-phase voltage $u_{s}$ of power grid (standard voltage) is converted into square-wave voltage $u_{\text {imp }}$ having the same frequency through the analogue comparator. Leading edges of square-wave voltage generate synchronizing pulses which initiate $\mathrm{S} \& \mathrm{H}$ circuit. The phase $2 \pi$ is compared with the actual value of the signal $\gamma$ generated by integrator. A quickness of $\gamma$ increase depends on an abruptly changing variable $u_{F}$ as the signal $\gamma$ comes from $u_{F}$ integration. Moreover, the synchronizing pulses reset integrator and thereupon signal $\gamma$ begins increasing again. The values of sine and cosine functions are read from look-up tables in microprocessor system for actual value $\gamma$. The values are used in order to approx- imate the voltage vector components $u_{s \alpha}=u_{s m} \cos \gamma$ and $u_{s \beta}=$ $=u_{s m} \sin \gamma$, whereas amplitude $u_{s m}$ is known.

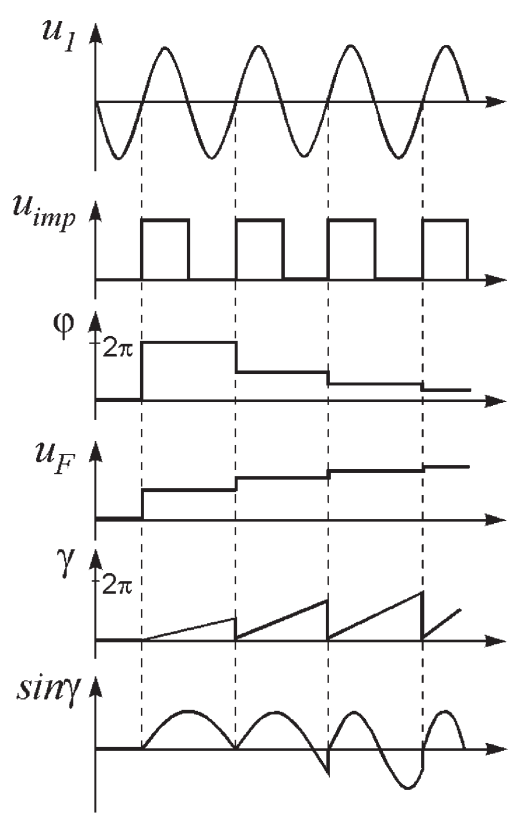

Fig. 9 Time-dependences in the system shown in Fig. 8

A discrete controller $\mathrm{C}$ provides integrator I with abruptly changing variable $u_{F}$ in determined intervals $\Delta t=t_{j}-t_{j-1}$ when synchronizing pulses occur in the system. Output signal of controller is changed in accordance to the following formula:

$$
u_{F(j)}=u_{F(j-1)}+k u_{\varphi(j)},
$$

where $u_{F(j)}, u_{F(j-1)}$ are values corresponding with actual and previous synchronizing pulse, respectively, $k$ is controller amplification.

The time-dependences explaining a principle of system operation are shown in Fig. 9. The variable $\gamma$ is constant if signal $u_{F}$ is equal to zero, i. e. the rest frequency of oscillator is also equal to zero. Even significant deviation of standard frequency from rest frequency is followed by synchronization of presented system. Thus, operating range of considered PLL is limited only by microprocessor parameters.

\section{Conclusion}

Two examples of application of phase locked loop in control system with double fed machine are presented in the paper. First example concerns synchronization of inverter current generated to the rotor winding depending on power grid frequency and rotor velocity. The second example concerns estimation of power grid voltage vector components. Comparing properties of proposed 
systems containing PLL and properties of systems known from radio engineering characterized in the first chapter of the paper, it may be concluded that:

1. In the PLL circuit using multiplier as phase detector the controlled variable is distorted. The low-pass filter is applied in order to minimize the distortion. It is a reason of slower operation of considered circuit. In the poly-phase systems, e. g. DFM control systems, the control of spatial vector position angle replaces synchronization of single-phase signals. A controlled variable proportional to angle is undistorted. Thus, filtration is unnecessary.

2. An application of PLL for synchronization of inverter current with power grid frequency and rotor velocity allows omitting the rotor position sensor in DFM control system. Properties of proposed by author DFM control system with PLL are similar to the system with encoder.

3. The pulse PLL used for estimation of power grid voltage components allows reducing a number of analogue inputs and simplifies meter circuits. Moreover, it possesses filtering properties and it does not cause a phase shift between standard (grid) voltage and estimated voltage.

4. Operating range of structures proposed by author is wider than those known from radio engineering and telecommunications.

\section{References}

[1] POPENDA, A.: Decoupling control of doubly fed induction machine without rotor position measurement, Proc. of Conf. PCIM'95, Nuremberg, 1995, pp. 561-571.

[2] POPENDA, A.: Control of double fed asynchronous machine using digital signal processor, Proc. of Conf. EDPE'99, High Tatras, 1999.

[3] TIETZE, U., SCHENK, CH.: Semiconductor Control Systems (in Polish), Warszawa WNT, 1997.

[4] KUDREWICZ, J.: Phasing loops dynamics (in Polish), Warszawa WNT, 1991.

[5] BOGALECKA, E.: Control problems with double-fed machine operating as a generator in electric power system (in Polish), Prace Naukowe Wyzszej Szkoly Morskiej w Gdyni, Gdynia, 1997.

[6] RUSEK, A., POPENDA, A.: Transient states of polymerizer drive including real load of specially designed induction motor, Proc. of Conf. on Electrical Machines (ICEM), Crete Island, 2006.

[7] RUSEK, A.: The mathematical model and selected transient states of polymerization reactor drive supplied with frequency converter, IV ${ }^{\text {th }}$ Int. Sc. Symp. Elektroenergetika 2007, 19.-21. 9. 2007, Stara Lesna, in press.

[8] POPENDA, A., RUSEK, A.: The impact of magnetic circuit saturation on properties of specially designed induction motor for polymerization reactor, ISEF 2007 - XIII Symposium on Electromagnetic Fields in Mechatronics, Electrical and Electronic Engineering, Prague, 2007, in press.

[9] POPENDA. A., RUSEK. A.: Mathematical model and selected transients states of main drive for polimerization reactor taking into consideration operating parameters of mixer chamber (in Polish), VI Sc. Symp. PTETiS "Wybrane Zagadnienie Elektrotechniki i Elektroniki (Selected Problems of Electrical and Electronic Engineering) WZEE'2006", Lublin - Kazimierz Dolny, 2006, pp. 220-229.

[10] POPLAWSKI, T.: Application of the Takagi-Sugeno (TS) fuzzy logic model for load curves prediction in the local power system, III ${ }^{\mathrm{rd}}$ Scientific Symposium Elektroenergetika 2005, Stara Lesna, 2005. 